case the healthy behaviours may prevent MS, and the geographical variation would need another explanation.

Demonstrating a causal link through a randomized controlled trial (RCT) of vitamin D would be complex, lengthy and costly, and such a trial may not be funded anyway because it would not test a drug. Richards' team tackled this problem by conducting a study that he calls "nature's RCT". They analysed large databases to find genetic variants that lowered vitamin $\mathrm{D}$ levels and then, by using another genetic database of people with MS, determined the MS risk in people with these variants. They found a strong risk of MS in people who have naturally low levels of vitamin D and the risk increased as the vitamin D level decreased ${ }^{10}$. "The person had their genetic make-up before they had MS. That's important, because the disease cannot influence the genes," he says.

The mounting evidence of the link with vitamin $\mathrm{D}$ has sparked discussion about how low levels could lead to MS. Bar-Or points out that vitamin $\mathrm{D}$ has been shown to affect the immune response in many of the cell types involved in MS, including myeloid, B and T cells. "There's an enormous amount of literature that associates vitamin $\mathrm{D}$ deficiency with risk of disease," adds Richards.

Røsjø thinks that this points to the connection with EBV. His research follows up a theory ${ }^{11}$ that vitamin $\mathrm{D}$ may boost immune responses to EBV and, conversely, that a lack of vitamin D may lead to poor responses to EBV. He thought that a weak immune response would increase levels of the biomarker EBNA1. "This in some way reflects increased risk of MS disease development," says Røsjø, although the molecular basis is still unknown. But in vitro studies have shown that the form of vitamin D that is active in the body, 1,25-dihydroxyvitamin D3, inhibits the production of antibodies from B cells, which are the cells infected by EBV.

Røsjø's study has borne out this theory. In a long-term placebo-controlled study of highdose vitamin D supplementation in people with MS, Røsjø found ${ }^{12}$ that levels of antibodies against EBNA1 fell significantly over the first 48 weeks of the study, but then rebounded, although never to their original levels, over the following 48 weeks. Røsjø says that this study was the first indication of an interaction between vitamin D and EBV. "There might be a short-term effect" of vitamin D on MS, he adds, "but it's lost over time."

The study also looked at two other viruses in the same herpesvirus family as EBV - varicella zoster virus, which causes chickenpox and shingles, and cytomegalovirus - and found that levels of antibodies to these viruses were unaffected by vitamin D. "There is something special, something unique with EBV that we cannot explain," Røsjø concludes.

Røsjø found that even large doses of vitamin D do not help patients with established MS in the long term, but he thinks that it may have a role during initial EBV infection. "Vitamin D and EBV during adolescence might be some of the most important things when it comes to deciding MS risk," he says.

\section{FAMILY FORTUNES}

One of the big puzzles about MS is that it runs in some families, but it can also pop up sporadically in people with no family history of the disease. Meagan McEwen, who campaigns for people with MS in Ottawa and has the disease, and her boyfriend, who also has it, embody this puzzle. "In my boyfriend's family, there are many other people who have MS. That's not true in my family," she says. This paradox has fuelled hundreds of studies worldwide.

One such study focused on the risk factors associated with the onset of MS in Canadian

\section{"The genetic contribution doesn't explain even half the risk."}

children. A team of researchers, including Bar-Or, investigated several major risk factors, including EBV, vitamin D and a specific variant of the $H L A-D R B 1$ gene, which is one of the human leukocyte antigen (HLA) complex of genes that are involved in the immune response ${ }^{13}$. "One of the benefits of studying children is that there's a relatively short time between the time they are born and the time they develop disease," explains Ruth Ann Marrie, a neurologist at the University of Manitoba in Winnipeg and one of the coauthors. "There is less time for either extraneous or relevant events to occur."

Bar-Or and Marrie found that the genetic variant most strongly associated with MS around the world, $H L A-D R B 1^{*} 15$, doubled the risk of children having MS. Since that study was published in 2011, the number of genetic variants known to affect MS risk has climbed from roughly 50 to about 200 .

Bar-Or stresses that none of these MSassociated variants is abnormal - they are not 'disease genes' like the mutations that cause cystic fibrosis, for example. "There are many, many more healthy people who have one or more combination of those very same variants than there are people who develop MS," he says. It seems that environmental factors or epigenetics - changes to gene expression that do not alter the genetic code - may explain the different outcomes, even for people with the same genetic make-up. "Two individuals who may have the identical template end up manifesting their genes in different ways based on epigenetics," says Bar-Or.

Genetic susceptibility can also vary geographically, Marrie points out, which may help to explain some clusters. A study of genetic risk factors for MS in Ireland, for example, showed that the percentage of individuals with the genetic variants that put them at risk of MS was substantially lower in one county than in another one nearby ${ }^{14}$. "The genetic contribution doesn't explain even half the risk," cautions Bar-Or, so it may be possible to prevent the disease by targeting the environmental factors involved.

Marrie thinks that the future direction of MS research will be into how these factors interact. "Now that we have identified multiple potential factors, we will work on the biology of the relationship to understand how these factors work together, and whether order of exposure is important," says Marrie. But she and others admit that definitively proving the causal role of any of these factors may be impractical or even impossible.

None of the researchers are ready to say what factors might be causing the Canadian clusters. Could it be the long, dark winters? A genetic variant common in French Canadians? A local EBV outbreak? Some combination of all three?

Dutrisac hopes that information about his cluster can help researchers to find the answer. "There's something out there. We need to find the cause," he says.

Carolyn Brown is a freelance writer based in Ottawa, Canada.

1. Torabi, M., Green, C., Yu, N. \& Marrie, R. A Neuroepidemiology 43, 38-48 (2014).

2. Multiple Sclerosis International Federation Atlas of MS (MSIF, 2013).

3. Poppe, A. Y., Wolfson, C. \& Zhu, B. Can. J. Neurol. Sci. 35, 593-601 (2008).

4. Beck, C. A., Metz, L. M., Svenson, L. W. \& Patten, S. B. Mult. Scler. 11, 516-519 (2005).

5. Warren, S. \& Warren, K. G. Neurology 43, 1760 1763 (1993).

6. Guimond, C. et al. Mult. Scler. 20, 1182-1188 (2014).

7. Orton, S.-M. et al. J. Neurol. Neurosurg. Psychiatry 81, 31-36 (2010).

8. Orton, S.-M. et al. Lancet Neurol. 5, 932-936 (2006).

9. Belbasis, L., Bellou, V., Evangelou, E., loannidis, J. P. A. \& Tzoulaki, I. Lancet Neurol. 14, 263-273 (2015).

10.Mokry, L. E. et al. PLoS Med. 12, e1001866 (2015).

11. Holmoy, T. Med. Hypoth. 70, 66-69 (2008).

12.Røsjø, E. et al. Mult. Scler. J. http://dx.doi.org/ $10.1177 / 1352458516654310$ (2016).

13.Banwell, B. et al. Lancet Neurol. 10, 436-445 (2011).

14.McGuigan, C. et al. J. Neurol. 252, 1245-1248 (2005). 